\title{
A NOTE ON ANALYTIC SETS
}

\author{
JOHN R. STEEL ${ }^{1}$
}

\begin{abstract}
We give "effective" proofs of two recent theorems on analytic sets of reals, together with counterexamples to their natural extensions.
\end{abstract}

In this note we give effective ("lightface") proofs of two recent theorems about analytic $\left(\Sigma_{1}^{1}\right)$ sets of reals due to $C$. Dellacherie, together with counterexamples to their natural extensions. All our proofs are quite easy modulo the basics of the effective theory.

In the case $k=2$, the following is just the Reduction Theorem for $\Pi_{1}^{1}$ sets of reals.

TheOREM 1 (DellacherIe [1]). Let $\left\{A_{i} \mid i<k\right\}$ be a finite family of $\Pi_{1}^{1}$ subsets of ${ }^{\omega} \omega$. Then there are $\Pi_{1}^{1}$ sets $B_{i}, i<k$, so that

(i) $B_{i} \subseteq A_{i}$ for $i<k$;

(ii) $B_{i} \cup B_{j}=A_{i} \cup A_{j}$ for $i \neq j$;

(iii) $\cap_{i<k} B_{i}=\varnothing$.

Proof. Let $R(i, x)$ iff $x \in A_{i}$. Let $\varphi: R \rightarrow \omega_{1}$ be a $\Pi_{1}^{1}$ norm on $R$ (cf. [3]) and put $\varphi(i, x)=\omega_{1}$ if $\neg R(i, x)$. Let

$$
x \in B_{i} \Leftrightarrow R(i, x) \wedge \exists j<k(\varphi(i, x)<\varphi(j, x) \vee(\varphi(i, x)=\varphi(j, x) \wedge i<j)) .
$$

Then $B_{i}$ is $\Pi_{1}^{1}$. Note that if $\exists i<k\left(x \notin A_{i}\right)$ then $\forall i<k\left(x \in A_{i} \Leftrightarrow x \in B_{i}\right)$, while if $\forall i<k\left(x \in A_{i}\right)$ then $x \notin B_{i}$ for exactly one $i<k$. Thus the $B_{i}$ are as desired.

Dellacherie asked whether Theorem 1 holds for countable families. A. S. Kechris and the author found a counterexample, which L. Harrington simplified considerably to the following. (Apparently, Kunen had earlier found a coupterexample using forcing.)

COUNTEREXAMPLE 1. Let

$$
\begin{aligned}
& x \in A_{0} \Leftrightarrow \text { codes an } \omega \text {-sequence }\left\{C_{n}^{x} \mid n<\omega\right\} \text { of } \Pi_{1}^{1} \text { subsets of }{ }^{\omega} \omega ; \\
& x \in A_{n+1} \Leftrightarrow x A_{0} \wedge x \in C_{n}^{x} .
\end{aligned}
$$

Suppose $\left\{B_{n} \mid n<\omega\right\}$ were an $\omega$-sequence of $\Pi_{1}^{1}$ sets reducing $\left\{A_{n} \mid n<\omega\right\}$ in the sense of Theorem 1. Let $x$ code $\left\{B_{n} \mid n<\omega\right\}$; i.e. let $B_{n}=C_{n}^{x}$ for $n<\omega$. By (iii) let $n$ be least so that $x \notin B_{n}$. Then $x \in A_{m}$ for $m<n$ by the definition of $\left\{A_{n} \mid n<\omega\right\}$. So $x \in B_{n+1}$ by (ii). But then $x \in A_{n+1}$, so $x \in C_{n}^{x}=B_{n}$, a contradiction.

Received by the editors April 14, 1979.

AMS (MOS) subject classifications (1970). Primary $02 K 30$.

${ }^{1}$ Research supported in part by NSF Grant No. MCS 78-02989. 
If $R \subseteq A \times B$ and $x \in A$, we let $R_{x}=\{y \in B \mid(x, y) \in R\} . R_{x}$ is called a section of $R$.

Theorem 2 (Dellacherie [1]). Let $R \subseteq{ }^{\omega} \omega \times{ }^{\omega} \omega$ be $\Sigma_{1}^{1}$, and $R_{x}$ compact for each $x \in{ }^{\omega} \omega$. Then there is a $\Sigma_{1}^{1}$ set $S \subseteq{ }^{\omega} \omega \times{ }^{\omega} \omega$ so that

(a) $\forall x\left(S_{x}\right.$ is compact and nonempty);

(b) $\forall x\left(R_{x} \neq \varnothing \Rightarrow R_{x}=S_{x}\right)$.

Proof. Let $R$ be as above. Define $R^{*}$ by

$$
R^{*}(x, s) \Leftrightarrow s \in \omega^{<\omega} \wedge \exists y(s \subseteq y \wedge R(x, y)) .
$$

Then $R^{*}$ is $\Sigma_{1}^{1}$, and $R_{x}^{*}$ is a tree on $\omega$ for each $x$. Since $R_{x}$ is closed, $R_{x}$ is just the set of branches of $R_{x}^{*}$; since $R_{x}$ is compact, $R_{x}^{*}$ is finitely branching. An easy boundedness argument yields a $\Delta_{1}^{1}$ set $T^{*} \supseteq R^{*}$ so that $T_{x}^{*}$ is an infinite, finitely branching tree on $\omega$ for each $x$.

Let $\varphi$ be a $\Pi_{1}^{1}$ norm on $\left({ }^{\omega} \omega \times \omega^{<\omega}\right)-R^{*}$ and define

$$
\psi(x, s)=\min \{\varphi(x, t) \mid t \subseteq s\} .
$$

Then $\psi$ is a $\Pi_{1}^{1}$ norm on $\left({ }^{\omega} \omega \times \omega^{<\omega}\right)-R^{*}$, and $\{s \mid \psi(x, s)>\alpha\}$ is a tree for each $x, \alpha$. Let

$$
S^{*}(x, s) \Leftrightarrow T^{*}(x, s) \wedge\left(R^{*}(x, s) \bigvee T_{x}^{*}-\{t \mid \psi(x, t)<\psi(x, s)\} \text { is finite }\right) .
$$

$S_{x}^{*}$ is a finitely branching tree for each $x$, and $S_{x}^{*}=R_{x}^{*}$ if $R_{x}^{*}$ is finite (i.e., if $\left.R_{x} \neq \varnothing\right)$. If $R_{x}^{*}$ is finite, let $\alpha$ be least so that $T_{x}^{*}-\{t \mid \psi(x, t)<\alpha\}$ is finite. Since $T_{x}^{*}$ is finitely branching, $\alpha=\beta+1$ for some $\beta$. But then $\psi(x, s)=\beta$ for infinitely many $s$, and $\psi(x, s)=\beta \Rightarrow S^{*}(x, s)$ by the definition of $S^{*}$. Thus $S_{x}^{*}$ is an infinite finitely branching tree for each $x$. Let $S(x, y) \Rightarrow y$ is a branch of $S_{x}^{*}$.

Dellacherie asked whether Theorem 2 holds with " $\sigma$-compact" replacing "compact".

Counterexample 2. Let $\left\{A_{n} \mid n<\omega\right\}$ be as defined in Counterexample 1. Let

$$
R(x, y) \Leftrightarrow \exists n\left(\forall i(y(i)=n) \wedge x \notin A_{n}\right) .
$$

Then $R$ is $\Sigma_{1}^{1}$ and $R_{x}$ is countable, hence $\sigma$-compact, for each $x$. The argument of Counterexample 1 shows that if $S \subseteq{ }^{\omega} \omega \times{ }^{\omega} \omega$ is $\Sigma_{1}^{1}$ and $\forall x\left(R_{x} \neq \varnothing \Rightarrow R_{x}=S_{x}\right)$, then $\exists x\left(R_{x}=\varnothing \wedge S_{x}=\varnothing\right)$.

The following is an old theorem.

TheOREM 3 (LUSIN [2]). Let $R \subseteq \subseteq^{\omega} \omega \times{ }^{\omega} \omega$ be $\Sigma_{1}^{1}$ and $R_{x}$ countable for each $x$. Then there are $\Sigma_{1}^{1}$ sets $S_{n}, n<\omega$, so that

(i) $R=\cup_{n} S_{n}$;

(ii) for each $x, n,\left(S_{n}\right)_{x}$ has $<1$ element.

Dellacherie asked whether Theorem 3 holds with " $\sigma$-compact" replacing "countable" and "is compact" replacing "has < 1 element".

CountereXAmple 3. For $x \in{ }^{\omega} \omega$, let $W^{x}=\{e \in \omega \mid e$ is the Gödel number of a well-order of $\omega$ recursive in $x$ \}. The relation " $y=W^{x}$ " is $\Pi_{1}^{1}$, as is " $y=W^{W^{x}}$. Define now

$$
R(x, y) \Leftrightarrow y \in{ }^{\omega} 2 \wedge y \neq W^{W^{x}}
$$


Then $R$ is $\Sigma_{1}^{1}$, and $R_{x}$ is $\sigma$-compact (in fact, open relative to ${ }^{\omega} 2$ ) for each $x$. Suppose $\left\{S_{n} \mid n<\omega\right\}$ were a family of $\Sigma_{1}^{1}$ sets as in the proposed extension of Theorem 3 . Say that " $x \in S_{n}$ " is a $\Sigma_{1}^{1}(z)$ relation of $x, n$. Let $S_{n}^{*}=\left\{s \in \omega^{<\omega} \mid \exists y(s \subseteq y \wedge y \in\right.$ $\left.\left.S_{n}\right)\right\}$. The sequence $\left\langle S_{n}^{*}\right| n\langle\omega\rangle$ is recursive in $W^{z}$. But $W^{W^{z}}$ is the unique $y \in{ }^{\omega} 2$ so that $y$ is not a branch of any of the trees $S_{n}^{*}$. Thus $W^{W^{z}}$ is $\Delta_{1}^{1}$ in $W^{z}$, a contradiction.

Counterexample 3 raises the following question: let $R \subseteq{ }^{\omega} \omega \times{ }^{\omega} \omega$ be $\Sigma_{1}^{1}$ with $\sigma$-compact sections. Must there be $S \subseteq R, \Sigma_{1}^{1}$ with compact sections, so that $R_{x} \neq \varnothing \Rightarrow S_{x} \neq \varnothing$ ? Clearly this is one instance of a family of problems generalizing the uniformization problem. If $R, S \subseteq{ }^{\omega} \omega \times{ }^{\omega} \omega$ we say $S$ almost uniformizes $R$ iff $S \subseteq R$ and $\forall x\left(R_{x} \neq \varnothing \Rightarrow S_{x} \neq \varnothing\right)$. Notice that if $R$ is $\Sigma_{1}^{1}$, then there is a $\Sigma_{1}^{1} S$ with Borel sections which almost uniformizes $R$; let $S x y \Leftrightarrow\left(R x y \wedge \omega_{1}^{x}=\omega_{1}^{x_{y} y}\right)$, where $\omega_{1}^{z}$ is the least ordinal not recursive in $z$. On the other hand, L. Harrington has found a $\Sigma_{1}^{1}$ relation $R$ such that, for every countable $\alpha, R$ cannot be almost uniformized by a $\Sigma_{1}^{1}$ relation with $\Pi_{\alpha}^{0}$ sections. We sketch his example for those familiar with [4]. Let $P$ be the forcing notion of $\$ 2$ of [4]. Let $R(x, T)$ iff $T$ is P-generic over $L_{\omega_{1}^{x}}[x]$ with respect to $\Sigma_{1}$ sentences. Then $R$ is $\Sigma_{1}^{1}$, since $R(x, T)$ iff $\omega_{1}^{x}=\omega_{1}^{x, T}$ and $T$ is P-generic over $L_{\omega_{1}^{x}}[x]$ with respect to ranked sentences. Lemma 1 of [4] implies that $R$ cannot be almost uniformized by a $\Sigma_{1}^{1}$ relation with sections of bounded Borel class.

\section{REFERENCES}

1. C. Dellacherie, Ensembles analytiques: théorèmes de séparation et applications, Séminaire de Probabilités IX, Lecture Notes in Math., vol. 465, Springer-Verlag, Berlin and New York, 1975.

2. N. Luzin, Lecons sur les ensembles analytiques et leurs applications, 2nd ed., Chelsea, New York, 1972.

3. Y. N. Moschovakis, Elementary incuction on abstract structures, North Holland, Amsterdam, 1974.

4. J. R. Steel, Forcing with tagged trees, Ann. Math. Logic 15 (1978), 55-74.

Department of Mathematics, University of California, los Angeles, California 90024 